Société Suisse de Microbiologie/Schweizerische

\title{
Mikrobiologische Gesellschaft
}

$15^{\mathrm{e}}$ assemblée annuelle, les 9 et 10 juin 1956, à Lausanne/15. Jahresversammlung, 9. und 10. Juni 1956, in Lausanne.

President - Präsident R. Regamey, Bern.

Viceprésident - VizepГäsident P. Kästli, Liebefeld-Bern.

Trésorier - Quästor Ch. Terrier, Neuchâtel.

ler Assesseur - 1. Beisitzer H. Mooser, Zurich.

2e Assesseur - 2. Beisitzer H. U. Gubler, Liebefeld-Bern.

ler jour : le 9 juin 1956. - 1. Tag: 9. Juni 1956.

Communications - Mitteilungen

Champendal, P. (Berne): Recherches bactériologiques à pro-

pos d'une épidémie hospitalière de staphylococcies . . 497

Zogg, H. (Zürich-Oerlikon): Uber die Beeinflussung von Pa-

thogenität und Wachstum eines pflanzlichen Parasiten

durch die Vitamine B1 und Biotin 501

Sansonnens, R. (Berne): Les suspensions homogènes de My-

cobactéries dans $\Gamma$ expérimentation in vitro et in vivo .504

Rívkíne, A. (Zurich): Influence de certains anions tensio-ac-

tifs sur les reactions d'agglutination spécifique étudiées

sur le syslème $\mathrm{T}$ des streptocoques hémolytiques de

groupe A 519

Beer, K. (Bern): Uber Torulose 534

Schmíd, G. (Bern): Über den Nachweis von Bang-Aggluti-

ninen im Vaginalsekret von Bang-infizierten Kühen . 539 Fey, H. (Zurich): Über Colistämme der O-Gruppe 78 mit

einem neuen B-Antigen bei der Colisepsis des Kalbes . 543 Py, G. (Neuchâtel): L'emploi des

filtres en microscopie et

microphotographie 551

Mooser, H., J. Lindenmann und F. Weyer (Zurich): Selek-

tiv-insektizide Wirkung des Butazolidin Geigy . . . 552

Vischer, W. A. (Davos): Eine Deckglasmethode zur länger-

dauernden kontinuierlichen Beobachtung von Zell- und

Bakterienkulturen .. 560

Tomcsik, J., und /. B. Baumann-Grace (Basel): Effekt me-

chanischer Einwirkungen auf die Zellwand gewisser Ba-

zillen 566

Pathologie und Bakterioíogie, Vol. 19, No. 5 (1956)

:ií) 
De Barbierí, A., et M. E. Scevola (Milano): Sư les modifica tions de Tactivité de quelques systèmes enzymatiques de Torganisme animal provoquées par $\Gamma$ antigène 0 et $\Gamma$ antigène $\mathrm{Vi} \quad 576$

Kellenberger, G., et E. (Geneve): Etude de souches colicinogènes au microscope électronique 582

Grasset, E., et B. Blondel (Geneve): Les formes L des Pasteurella : Facteurs engendrant leurs transformations . 598 Lavillaureix, J. (Strasbourg): Les formes L des vibrions : Leur pouvoir pathogène $\quad 615$ Kanítz, St., und P. Crovari (Genua): Erste Nachrichten über in Ligurien gefundene Coxsackie-Viren 620 Agnese, G. (Genes): Recherches suy les porteurs de virus poliomyélitique 624

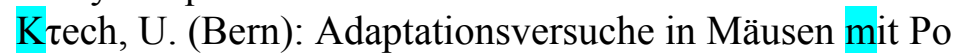
liomyelitis-Virus Typ $1 \quad 628$

Tomelliní, R. (Genes): Sur la frequence des anticorps neutralisant les trois types de virus poliomyélitique en Ligurie 633

Schär, B. (Basel): Vortrag zum Film «Leukozytotaktische Wirkung von Bakterienpolysacchariden» .... 637

2e jour: le 10 juin 1956. - 2. Tag: 10. Juni 1956.

Desaulles, P., B. Schär und R. Meier (Basel): Wirkung von Anthraxfraktionen auf mesenchymale Zellreaktionen in vitro und in vivo $\quad 639$ Schopfer, W. H. (Berne), et Th. Postemak (Geneve): Re cherches sur les anti-inositols. Action de Tisomytilitol sur Schizosaccharomyces Pombe (Lindner) souche liquefaciens (Osterwalder) Dekker 647

Recherches sur la biochimie des cyclitols. L'utilisation de trois inososes par six microorganismes . . 655 -, et F. Knüsel (Berne): Recherches sur la biosynthèse de la lactoflavine. Action du Zinc sur la biosynthèse de cette vitamine 659

ler Rapport principal - 1. Hauptvortrag Buchne $\tau$, P. (Ischia): Die harmonische Einbürgerung pflanzlicher Mikroorganismen in den tierischen Körper 664 2e Rapport principal - 2. Hauptvortrag Koch, A. (München): Die experimentelle Analyse der Bedeutung der Symbionten 665 